\section{Geometric Cooperative Control of Particle Formations}

\author{
Fumin Zhang
}

\begin{abstract}
We present a geometric approach for formation control that explicitly decouples translation dynamics from the orientation and shape dynamics. The formation dynamics are modeled as controlled Lagrangian systems on Jacobi shape space, and measurements of shape variables are used as feedback to control the entire formation. This geometric approach allows each member of the formation, modeled as a Newtonian particle, freedom to choose different coordinate frame and shape variables to describe observed orientation and shape of the formation. We derive a class of cooperative control laws and shape consensus algorithms with provable convergence. They can be implemented in a distributed fashion thanks to gauge covariance and coordinate independence associated with the geometric approach.
\end{abstract}

\section{INTRODUCTION}

Recent developments in control theory and robotics research have enabled cooperative missions performed by multiple mobile robots. The goal of formation control is to design distributed motion control and planning algorithms to achieve desired cluster shape. A large number of results have emerged in control theory regarding formation control and the closely related consensus problem [1], [2]. Typically, the motion dynamics of individual robot are simplified to a second order particle model. Graph theory is widely used to model the information exchange among particles [3]-[6].

In this paper, we introduce a geometric approach for formation control. After modeling the particle formation as a deformable body, we apply geometric reduction theory to explicitly decompose the collective motion dynamics of all particles into dynamics for the center, the orientation, and the shape of the deformable body. Described using the Jacobi shape coordinates [7], formation shape is invariant under translation and rotation, and is also independent of the coordinate system in which one chooses to describe it. The geometric approach allows each individual robot to establish a possibly different body frame and use different shape coordinates to observe and describe the formation without knowing the choices of body frame and shape coordinates of other robots. Freedom of choosing coordinates often arises when a certain coordinate system is more convenient than others towards getting more accurate estimates of the orientation and shape of the formation from sensor data. The geometric approach enables formation control laws that achieve shape consensus but do not achieve common velocity among particles, allowing more freedom in designing cooperative missions where the rotation of the formation is necessary. Our previous works [8], [9] and related work [10], [11] by other researchers applied shape theory to formation control. But shape dynamics and gauge covariance, together with their implications for formation control and consensus, are not treated in [10], [11]. This paper develops new convergence results on formation control that take advantage of gauge covariance and freedom of choosing shape variables.

\section{JACOBI SHAPE SPACE}

In this section, we introduce the Jacobi shape space that can be studied as a principal fiber bundle in geometric mechanics [12], [13].

F. Zhang is with School of Electrical and Computer Engineering, Georgia Institute of Technology, Savannah, GA 31407. Email: fumin@ece.gatech.edu.

The author thanks Prof. P. S. Krishnaprasad for his guidance and collaboration on this work, thanks Dr. E. W. Justh and Prof. N. E. Leonard for discussions and suggestions, and thanks the reviewers for constructive criticism. This research was supported in part by ONR grants N00014-081-1007 and N00014-09-1-1074, and by NSF grants ECCS-0841195, ECCS0845333(CAREER), and CNS-0931576.
Jacobi coordinates and Jacobi shape coordinates are introduced as local coordinates describing the principal bundle.

\section{A. The Jacobi Vectors}

In the inertial coordinate frame, let $q_{i} \in \mathscr{R}^{3}$, where $i=1,2, \ldots, N$, denote the coordinates of $N$ particles with masses $m_{i}$. Let $M=$ $\sum_{i=1}^{N} m_{i}$. Then the center of mass is $q_{c}=\frac{\sum_{i=1}^{N} m_{i} q_{i}}{M}$. We see that $q_{c}$ only describes the position of the entire formation and does not affect the formation shape or orientation. We seek $(N-1)$ independent vectors $\left(\rho_{\mathrm{f} i}, i=1,2, \ldots, N-1\right)$ such that the kinetic energy of the cluster, originally expressed as $K^{t o t}=\frac{1}{2} \sum_{i=1}^{N} m_{i}\left\|\dot{q}_{i}\right\|^{2}$, is block diagonalized as $K^{t o t}=\frac{1}{2} M\left\|\dot{q}_{c}\right\|^{2}+\frac{1}{2} \sum_{j=1}^{N-1}\left\|\dot{\rho}_{\mathrm{f} j}\right\|^{2}$. Such a set of $\rho_{\mathrm{f} j}$ are called Jacobi coordinates or Jacobi vectors. The definition of Jacobi vectors requires finding a special transform $\Phi$ from $q_{i}, i=1,2, \ldots, N$, to $\left(q_{c}, \rho_{\mathrm{f} j}\right), j=1,2, \ldots, N-1$. If we express $\Phi$ as a $(N \times N)$ matrix, then:

$$
\left[\begin{array}{llll}
q_{c} & \rho_{\mathrm{f} 1} & \ldots & \rho_{\mathrm{f}(N-1)}
\end{array}\right]=\left[\begin{array}{llll}
q_{1} & q_{2} & \ldots & q_{N}
\end{array}\right] \Phi .
$$

There is more than one way to construct the Jacobi coordinates. In general, between any two sets of Jacobi coordinates there exists an element $\mathbf{h} \in \mathrm{O}(\mathrm{N}-1)$, i.e. the orthogonal group, such that $\left[\begin{array}{llll}\rho_{\mathrm{f} 1}^{1} & \rho_{\mathrm{f} 2}^{1} & \ldots & \rho_{\mathrm{f}(N-1)}^{1}\end{array}\right]=\left[\begin{array}{llll}\rho_{\mathrm{f} 1}^{2} & \rho_{\mathrm{f} 2}^{2} & \ldots & \rho_{\mathrm{f}(N-1)}^{2}\end{array}\right] \mathbf{h}$. This orthogonal group $\mathrm{O}(\mathrm{N}-1)$ is called the democracy group [7].

Let $\mathscr{R}^{3 N}$ be the total configuration space of a formation of $N$ particles in 3D space. After the center coordinates are removed, the space of Jacobi coordinates is $\mathscr{R}^{3 N-3}$. Since the shape of the formation is independent of its orientation, we can view this symmetry as induced by the rigid rotation group $\mathrm{SO}(3)$ acting on $\mathscr{R}^{3 N-3}$ on the left as $g \rho_{\mathrm{f} j}$ for $g \in \mathrm{SO}(3)$ and $j=1,2, \ldots, N-1$. This action is proper and free except for the shapes where all $\rho_{\mathrm{f} j}$ are collinear, i.e., all particles are on a straight line. We let the set $F_{0}$ be the set of all the Jacobi coordinates corresponding to collinear shapes. Let $F=\mathscr{R}^{3 N-3}-F_{0}$ and call it the Jacobi pre-shape space. It is an open sub-manifold of $\mathscr{R}^{3 N-3}$. Since $\mathrm{SO}(3)$ acts properly and freely on $F$, the base space $B=F / \mathrm{SO}(3)$ is a smooth manifold and the canonical projection $\pi: F \rightarrow B$ is differentiable. $B$ is called the Jacobi shape space.

In dropping from $F$ to $B$, we remove the rotational symmetry from the Jacobi coordinates. After the reduction, the dimension of the shape space $B$ is $(3 N-6)$. Therefore, we need $3 N-6$ variables to describe a rigid formation in three dimensional space. On this shape space we can define shape variables $s_{k}$ for $k=1,2, \ldots,(3 N-6)$ which are rigid motion invariant. Mutual distances, mutual angles, areas and volumes formed by the line segments connecting the particles all serve as candidates for shape variables. The tuple $(F, B, \operatorname{SO}(3), \pi)$ formulate a principal fiber bundle. For each point $\mathbf{s}$ in the Jacobi shape space, the fiber is homeomorphic to $\mathrm{SO}(3)$.

\section{B. Gauge Conventions and Gauge Transforms}

For a given shape $\mathbf{s}=\left(s_{1}, s_{2}, \ldots, s_{3 N-6}\right)$, we can measure its orientation by attaching a body coordinate system to the formation, and then obtain a group element $g \in \mathrm{SO}(3)$ as the result. The Jacobi coordinates $\rho_{j}$ in the body coordinate system are related to the Jacobi coordinates $\rho_{\mathrm{f} j}$ in the inertial frame by $\rho_{\mathrm{f} j}=g \rho_{j}(\mathbf{s})$ for $j=1,2, \ldots, N-1$. Hence the $\rho_{j}$ are vector valued functions of the shape variables $\mathbf{s}$ only.

When the shape of the formation changes, the body coordinate system should be consistent i.e. the procedure to establish a body coordinate system should be shape independent. Such a shape independent procedure for establishing a body coordinate system is called a gauge convention [7]. Formally, a gauge convention is a 
diffeomorphism between $F$ and $\mathrm{SO}(3) \times B$ mapping any point $\rho_{\mathrm{f}} \in F$ to $(g, \mathbf{s}) \in \mathrm{SO}(3) \times B$ such that $g_{1} \boldsymbol{\rho}_{\mathrm{f}} \mapsto\left(g_{1} g, \mathbf{s}\right)$ for all $g_{1} \in \mathrm{SO}(3)$.

Let $g \in \mathrm{SO}(3)$ describe the orientation of the formation for any shape $\mathbf{s}$ under one gauge convention. Let $g^{1} \in \mathrm{SO}(3)$ describe the orientation of the formation for the same $\mathbf{s}$ under another gauge convention. Then by the property of $\mathrm{SO}(3)$, there exists $h(\mathbf{s})$ such that $g=g^{1} h^{\top}(\mathbf{s})$ where $h: B \rightarrow \mathrm{SO}(3)$ is a $\mathrm{SO}(3)$ valued function on $B$. This right action of $h(\mathbf{s})$ on $\mathrm{SO}(3)$ is called a gauge transform. Because a gauge transform is a shape dependent group action, an object that obeys simple transformation rules under rigid group action by $\mathrm{SO}(3)$ may violate such rules under a gauge transform. We say an object is gauge invariant if it is invariant under any gauge transform. We say an object is gauge covariant if it obeys the transformation rules for rigid group action by $\mathrm{SO}(3)$ when it is subjected to a gauge transform. An example of a gauge invariant object is the collection of shape variables $\mathbf{s}$. An example of gauge covariant objects are the Jacobi vectors $\rho_{j}$ in the body frame for $j=1,2, \ldots, N-1$, since if $g \rho_{j}=\rho_{\mathrm{f} j}=g^{1} \rho_{j}^{1}$, we have $\rho_{j}=h(\mathbf{s}) \rho_{j}^{1}$. Hence $\rho_{j}^{1}$ are transformed to $\rho_{j}$ via the rigid left action by $h(\mathbf{s})$.

Some mechanical quantities can be defined in the body frame, which are associated with the Kinetic energy $K=\frac{1}{2} \sum_{j=1}^{N-1}\left\|\dot{\rho}_{\mathrm{f} j}\right\|^{2}$. We define $\Omega$ to be the angular velocity that satisfies $\dot{g}=g \widehat{\Omega}$ where $\widehat{\Omega}$ is the $3 \times 3$ skew symmetric matrix created from $\Omega$ such that $\Omega \times \mathbf{x}=\widehat{\Omega} \mathbf{x}$ for any vector $\mathbf{x}$. Then $\dot{\rho}_{\mathrm{f} j}=g\left(\Omega \times \rho_{j}+\sum_{k=1}^{3 N-6} \frac{\partial \rho_{j}}{\partial s_{k}} \dot{s}_{k}\right)$. Letting $\mathbf{e}$ be the $3 \times 3$ identity matrix, we define $I(\mathbf{s})=\sum_{j=1}^{N-1}\left(\left\|\rho_{j}\right\|^{2} \mathbf{e}-\rho_{j} \rho_{j}^{\top}\right)$ as the locked inertia tensor of the formation in the body coordinate frame. We also define the gauge potentials to be $A_{k}(\mathbf{s})=I^{-1} \sum_{j=1}^{N-1} \rho_{j} \times \frac{\partial \rho_{j}}{\partial s_{k}}$. Let $\mathbf{A}=\left[\begin{array}{llll}A_{1} & A_{2} & \ldots & A_{3 N-6}\end{array}\right]$ and define the shape metric tensor $G$ as $G_{k l}=-A_{k}^{\top} I A_{l}+\sum_{j=1}^{N-1} \frac{\partial \rho_{j}^{\top}}{\partial s_{k}} \frac{\partial \rho_{j}}{\partial s_{l}}$ for $k, l=1,2, \ldots, 3 N-6$. These quantities are defined on the shape space, i.e. they are independent of orientation, because $\rho_{j}$ only depend on shape variables. We can now rewrite the kinetic energy $K$ in block diagonalized form as

$$
K=\frac{1}{2}(\Omega+\mathbf{A} \dot{\mathbf{s}})^{\top} I(\Omega+\mathbf{A} \dot{\mathbf{s}})+\frac{1}{2} \dot{\mathbf{s}}^{\top} G \dot{\mathbf{s}} .
$$

The angular velocity $\Omega$ is not gauge covariant. For gauge covariance, we define a combined angular velocity as $\Upsilon=\Omega+\sum_{k=1}^{3 N-6} A_{k} \dot{s}_{k}$. We want to show that $\Upsilon$ is gauge covariant.

We define $\widehat{\gamma}_{k}=h^{\top} \frac{\partial h}{\partial s_{k}}$ for $k=1,2, \ldots, 3 N-6$. It is easy to see that $\widehat{\gamma}_{k}$ is in the Lie algebra so(3). Therefore, we can let $\gamma_{k}$ denote the vector representation of $\widehat{\gamma}_{k}$. We prove the following lemmas.

Lemma 2.1: Under the gauge transform $g=g^{1} h^{\top}(\mathbf{s})$, we have, for $k=1,2, \ldots, 3 N-6, A_{k}=h(\mathbf{s})\left(A_{k}^{1}+\gamma_{k}\right)$ and $I(\mathbf{s})=h(\mathbf{s}) I^{1}(\mathbf{s}) h^{\top}(\mathbf{s})$.

Proof: Under the gauge transform, the Jacobi vectors are transformed as $\rho_{j}=h(\mathbf{s}) \rho_{j}^{1}$ for $j=1,2, \ldots, N-1$. Therefore, according to the definition of the locked inertia tensor, $I(\mathbf{s})=h(\mathbf{s}) I^{1}(\mathbf{s}) h^{\top}(\mathbf{s})$ is true, i.e. the locked inertia tensor is gauge covariant. Using the definition for gauge potentials, for $k=1,2, \ldots, 3 N-6$, we have

$$
\begin{aligned}
A_{k} & =I^{-1} \sum_{j=1}^{N-1}\left(h(\mathbf{s}) \rho_{j}^{1}\right) \times \frac{\partial\left(h(\mathbf{s}) \rho_{j}^{1}\right)}{\partial s_{k}} \\
& =h(\mathbf{s})\left(I^{1}\right)^{-1} h^{\top}(\mathbf{s}) h(\mathbf{s}) \sum_{j=1}^{N-1} \rho_{j}^{1} \times\left(\frac{\partial \rho_{j}^{1}}{\partial s_{k}}+h^{\top}(\mathbf{s}) \frac{\partial h(\mathbf{s})}{\partial s_{k}} \rho_{j}^{1}\right) \\
& =h(\mathbf{s})\left(A_{k}^{1}+\left(I^{1}\right)^{-1} \sum_{j=1}^{N-1} \rho_{j}^{1} \times\left(\gamma_{k} \times \rho_{j}^{1}\right)\right) \\
& =h(\mathbf{s})\left(A_{k}^{1}+\left(I^{1}\right)^{-1} \sum_{j=1}^{N-1}\left(\left\|\rho_{j}^{1}\right\|^{2} \mathbf{e}-\rho_{j}^{1}\left(\rho_{j}^{1}\right)^{\top}\right) \gamma_{k}\right) \\
& =h(\mathbf{s})\left(A_{k}^{1}+\gamma_{k}\right) .
\end{aligned}
$$

Lemma 2.2: $\Upsilon$ is gauge covariant, i.e. $\Upsilon=h(\mathbf{s}) \Upsilon^{1}$ under the gauge transform $g=g^{1} h^{\top}(\mathbf{s})$.

Proof: We can calculate the transform of $\widehat{\Omega}$ as $\widehat{\Omega}=g^{-1} \dot{g}=$ $h(\mathbf{s})\left(\widehat{\Omega}^{1}-\sum_{k=1}^{3 N-6} \widehat{\gamma}_{k} \dot{s}_{k}\right) h^{\top}(\mathbf{s})$ Therefore, applying Lemma 2.1 , we have

$$
\begin{aligned}
\Upsilon & =\Omega+\sum_{k=1}^{3 N-6} A_{k} \dot{s}_{k} \\
& =h(\mathbf{s})\left(\Omega^{1}-\sum_{k=1}^{3 N-6} \gamma_{k} \dot{s}_{k}\right)+\sum_{k=1}^{3 N-6} h(\mathbf{s})\left(A_{k}^{1}+\gamma_{k}\right) \dot{s}_{k} \\
& =h(\mathbf{s}) \Upsilon^{1} .
\end{aligned}
$$

\section{Controlled Lagrange EQUations}

In [14] and [15], the Lagrange-D'Alembert principle for rigid body dynamics is rigorously formulated. In this section, we follow a similar approach to derive the dynamics for the formation viewed as a deformable body.

Some specialized notations are used in the derivations. The notation $\langle$,$\rangle represents the inner product between two column vectors, and$ the notation $\langle,\rangle_{\mathrm{M}}$ is the inner product between matrices defined as $\langle A, B\rangle_{\mathrm{M}}=\frac{1}{2} \operatorname{tr}\left(A^{\top} B\right)$ for arbitrary $A$ and $B$ with proper dimensions. Third order tensors will appear in the derivations. We introduce a bracketing notation $T[\mathbf{a}, \mathbf{b}, \mathbf{c}]_{\tau}$ to mean the third order tensor $T$ acts on three arbitrary vectors $\mathbf{a}, \mathbf{b}$, and $\mathbf{c}$, and the result is a scalar. The notation $T[\mathbf{a}, \mathbf{b}]_{\tau}$ means that the tensor $T$ acts on two vectors $\mathbf{a}$ and $\mathbf{b}$, and the result is a column vector such that $\mathbf{c}^{\top} T[\mathbf{a}, \mathbf{b}]_{\tau}=T[\mathbf{a}, \mathbf{b}, \mathbf{c}]_{\tau}$. Another relevant notation is $T^{*}$ that is the cyclic transpose of $T$ i.e. $T^{*}[\mathbf{a}, \mathbf{b}, \mathbf{c}]_{\tau}=T[\mathbf{b}, \mathbf{c}, \mathbf{a}]_{\tau}$ or $T_{i j k}^{*}=T_{j k i}$ where $i, j, k$ are indices for the third order tensors, c.f. [14] and [16]. We also use the notation $\widehat{(} \widehat{)}$ to denote the skew symmetric matrix representation of a three dimensional vector resulting from a long expression inside the parentheses.

\section{A. The Formation Dynamics}

In the lab frame, the particles satisfy the Newton's equations: $m_{i} \ddot{q}_{i}=\mathbf{f}_{i}$ for $i=1,2, \ldots, N$. Hence, the center of the formation satisfies the Newton's equation $M \ddot{q}_{c}=u_{c}$ where $u_{c}$ is the equivalent force applied to the center. On the Jacobi pre-shape space endowed with the Jacobi coordinates $\rho_{\mathrm{f} j}$, the system equations in the Jacobi coordinates are $\ddot{\rho}_{\mathrm{f} j}=u_{\mathrm{f} j}$ for $j=1,2, \ldots N-1$. We notice that the control forces $\mathbf{f}_{i}$ are combined into controls $u_{c}$ and $u_{\mathrm{f} j}$. For the choice of Jacobi coordinates given by equation (1), we can calculate the relations between the forces as $\left[\begin{array}{lllll}u_{c} & u_{\mathrm{f} 1} & \ldots & u_{\mathrm{f}(N-1)}\end{array}\right]=\left[\begin{array}{llll}\mathrm{f}_{1} & \mathrm{f}_{2} & \ldots & \mathrm{f}_{N}\end{array}\right] \Phi$.

We now go one step further to view the Jacobi pre-shape space $F$ as a principal fiber bundle with coordinates $(g, \mathbf{s})$ where $g \in \mathrm{SO}(3)$ and $\mathbf{s}=\left[\begin{array}{llll}s_{1} & s_{2} & \ldots & s_{3 N-6}\end{array}\right]^{\top} \in B$ where $B$ is the Jacobi shape space. A point in the tangent bundle $T F$ of $F$ can be represented by $(g, \mathbf{s}, g \widehat{\Omega}, \dot{\mathbf{s}})$ where $\widehat{\Omega} \in \operatorname{so}(3)$. The kinetic energy $K$ now has the form in equation (2) and the Lagrangian $L=K$. The Lagrange-D'Alembert principle states that $\frac{d}{d t}\left(D_{g \widehat{\Omega}} L, D_{\dot{\mathbf{s}}} L\right)-\left(D_{g} L, D_{\mathbf{s}} L\right)=\left(g u_{g}, u_{s}\right)$ where the symbol $D_{(\cdot)} L$ represents the differential of $L$ with respect to the variables within the parentheses, $u_{g}$ represents control effort on the orientation, and $u_{s}$ is the control effort on the shape.

To derive the equations for the dynamics we need to first compute the differentials $D L=\left(D_{g} L, D_{\mathbf{s}} L, D_{g \widehat{\Omega}} L, D_{\mathbf{S}} L\right)$. This is defined in terms of the Frechet derivative, if $Y$ is a displacement on $(g, \mathbf{s}, g \widehat{\Omega}, \dot{\mathbf{s}})$, then we say that, for any $\varepsilon$,

$$
D L(Y)=\left.\frac{d}{d \varepsilon}\right|_{\varepsilon=0} L((g, \mathbf{s}, g \widehat{\Omega}, \dot{\mathbf{s}})+\varepsilon Y) .
$$


It can be shown that the displacement $Y$ on the Jacobi shape space contains four components $Y=\left(g \widehat{\Omega}_{1}, \mathbf{v}_{1}, g\left(\widehat{\Omega}_{1} \widehat{\Omega}+\widehat{\Omega}_{2}\right), \mathbf{v}_{2}\right)$. We compute the left hand side of equation (5) as

$$
\begin{aligned}
D L(Y) & =\left\langle D_{g} L, g \widehat{\Omega}_{1}\right\rangle_{\mathrm{M}}+\left\langle D_{\mathbf{s}} L, \mathbf{v}_{1}\right\rangle \\
& +\left\langle D_{g \widehat{\Omega}} L, g\left(\widehat{\Omega}_{1} \widehat{\Omega}+\widehat{\Omega}_{2}\right)\right\rangle_{\mathrm{M}}+\left\langle D_{\dot{\mathbf{s}}} L, \mathbf{v}_{2}\right\rangle .
\end{aligned}
$$

We then compute the right hand side of equation (5) as

$$
\begin{aligned}
\left.\frac{d}{d \varepsilon} L\right|_{\varepsilon=0} & =\Omega_{2}^{\top} I(\Omega+\mathbf{A} \dot{\mathbf{s}})+\left(\frac{\partial \mathbf{A}}{\partial \mathbf{s}}\left[\mathbf{v}_{1}, \dot{\mathbf{s}}\right]_{\tau}\right)^{\top} I(\Omega+\mathbf{A} \dot{\mathbf{s}}) \\
& +\frac{1}{2} \frac{\partial I}{\partial \mathbf{s}}\left[\mathbf{v}_{1}, \Omega+\mathbf{A} \dot{\mathbf{s}}, \Omega+\mathbf{A} \dot{\mathbf{s}}\right]_{\tau} \\
& +\left(\mathbf{A v}_{2}\right)^{\top} I(\Omega+\mathbf{A} \dot{\mathbf{s}})+\frac{1}{2} \frac{\partial G}{\partial \mathbf{s}}\left[\mathbf{v}_{1}, \dot{\mathbf{s}}, \dot{\mathbf{s}}\right]_{\tau}+\mathbf{v}_{2}^{\top} G \dot{\mathbf{s}}
\end{aligned}
$$

Comparing equation (6) and (7), we first notice that there is only one term in each equation involving $\Omega_{2}$. This yields $D_{g \widehat{\Omega}} L=\widehat{g(I}(\Omega+$ $\mathbf{A} \dot{\mathbf{s}}) \widehat{)}$. Next, there is no term involving $\Omega_{1}$ in (7). From this we can find $D_{g} L=\widehat{g(I}(\Omega+\mathbf{A} \dot{\mathbf{s}}) \widehat{)} \widehat{\Omega}$. Observing the terms involving $\mathbf{v}_{2}$ in (7), we obtain $D_{\dot{\mathbf{s}}} L=\mathbf{A}^{\top} I(\Omega+\mathbf{A} \dot{\mathbf{s}})+G \dot{\mathbf{s}}$. Finally, we gather the terms in (7) that contain $\mathbf{v}_{1}$ and obtain

$$
\begin{aligned}
\left\langle D_{\mathbf{s}} L, \mathbf{v}_{1}\right\rangle & =\left(\frac{\partial \mathbf{A}}{\partial \mathbf{s}}\left[\mathbf{v}_{1}, \dot{\mathbf{s}}\right] \tau\right)^{\top} I(\Omega+\mathbf{A} \dot{\mathbf{s}}) \\
& +\frac{1}{2} \frac{\partial I}{\partial \mathbf{s}}\left[\mathbf{v}_{1}, \Omega+\mathbf{A} \dot{\mathbf{s}}, \Omega+\mathbf{A} \dot{\mathbf{s}}\right]_{\tau}+\frac{1}{2} \frac{\partial G}{\partial \mathbf{s}}\left[\mathbf{v}_{1}, \dot{\mathbf{s}}, \dot{\mathbf{s}}\right]_{\tau}
\end{aligned}
$$

In this equation $\frac{\partial \mathbf{A}}{\partial \mathbf{s}}, \frac{\partial I}{\partial \mathbf{s}}$ and $\frac{\partial G}{\partial \mathbf{s}}$ are third order tensors. We have

$$
\begin{aligned}
\left(\frac{\partial \mathbf{A}}{\partial \mathbf{s}}\left[\mathbf{v}_{1}, \dot{\mathbf{s}}\right]_{\tau}\right)^{\top} I(\Omega+\mathbf{A} \dot{\mathbf{s}}) & =\frac{\partial \mathbf{A}}{\partial \mathbf{s}}\left[\mathbf{v}_{1}, \dot{\mathbf{s}}, I(\Omega+\mathbf{A} \dot{\mathbf{s}})\right]_{\tau} \\
& =\left(\frac{\partial \mathbf{A}}{\partial \mathbf{s}}\right)^{*}\left[\dot{\mathbf{s}}, I(\Omega+\mathbf{A} \dot{\mathbf{s}}), \mathbf{v}_{1}\right]_{\tau}
\end{aligned}
$$

where $\left(\frac{\partial \mathbf{A}}{\partial \mathbf{s}}\right)^{*}$ is the cyclic transpose of $\frac{\partial \mathbf{A}}{\partial \mathbf{s}}$ as mentioned before. Therefore, we can solve for $D_{\mathrm{s}} L$ as

$$
\begin{aligned}
D_{\mathbf{s}} L & =\left(\frac{\partial \mathbf{A}}{\partial \mathbf{s}}\right)^{*}[\dot{\mathbf{s}}, I(\Omega+\mathbf{A} \dot{\mathbf{s}})]_{\tau}+\frac{1}{2}\left(\frac{\partial I}{\partial \mathbf{s}}\right)^{*}[\Omega+\mathbf{A} \dot{\mathbf{s}}, \Omega+\mathbf{A} \dot{\mathbf{s}}]_{\tau} \\
& +\frac{1}{2}\left(\frac{\partial G}{\partial \mathbf{s}}\right)^{*}[\dot{\mathbf{s}}, \dot{\mathbf{s}}]_{\tau} .
\end{aligned}
$$

Therefore, by applying the Lagrange-D'Alembert principle and then rearranging certain terms, we have proved the following theorem.

Theorem 3.1: The Lagrange equations of the formation dynamics are given by

$$
\begin{aligned}
\frac{d}{d t}(\Pi)=-(\Upsilon & -\mathbf{A} \dot{\mathbf{s}}) \times I+u_{g} \\
\frac{d}{d t}(G \dot{\mathbf{s}})+\mathbf{A}^{\top} \frac{d}{d t}(\Upsilon) & =\frac{1}{2}\left(\frac{\partial I}{\partial \mathbf{s}}\right)^{*}[\Upsilon, \Upsilon]_{\tau} \\
& +\left(\left(\frac{\partial \mathbf{A}}{\partial \mathbf{s}}\right)^{*}-\frac{\partial \mathbf{A}}{\partial \mathbf{s}}\right)[\dot{\mathbf{s}}, \Pi \Upsilon]_{\tau} \\
& +\frac{1}{2}\left(\frac{\partial G}{\partial \mathbf{s}}\right)^{*}[\dot{\mathbf{s}}, \dot{\mathbf{s}}]_{\tau}+u_{s}
\end{aligned}
$$

If the potential energy of the system is not zero, then terms involving the potential function enter the dynamics in simple manner as shown in [8], [9]. We have also shown that $u_{g}$ and $u_{s}$ are related to $u_{\mathrm{f} j}$ for $j=1,2, \ldots, N-1$ by $u_{g}=\sum_{j=1}^{N-1} \rho_{j} \times u_{j}$ and $u_{s k}=\sum_{j=1}^{N-1}\left(\frac{\partial \rho_{j}}{\partial s_{k}}\right)^{\top} u_{j}$ where $k=1,2, \ldots,(3 N-6)$ and $u_{j}=g^{-1} u_{\mathrm{f} j}$.

\section{B. Gauge Covariant Control Form}

Controllers can be synthesized by designing $\left(u_{g}, u_{s}\right)$ first and then computing $\mathbf{f}_{i}$ for $i=1,2, \ldots, N$, which are the actual force on each particle. This procedure is gauge dependent. We show that by transforming $\left(u_{g}, u_{s}\right)$ into a gauge covariant form, we can establish a controller design procedure that allows each particle to use its own gauge convention.

We define the gauge covariant cooperative control form to be the pair $\left(\alpha_{g}, \alpha_{s}\right)$ where $\alpha_{g}$ and the $k$ th component of $\alpha_{s}$ satisfy

$$
\begin{aligned}
\alpha_{g} & =u_{g} \\
\alpha_{s k} & =u_{s k}-<u_{g}, A_{k}>
\end{aligned}
$$

for $k=1,2, \ldots, 3 N-6$.

Lemma 3.2: The control $\left(\alpha_{g}, \alpha_{s}\right)$ defined by (13) are gauge covariant. Under the gauge transform $g=g^{1} h^{\top}(\mathbf{s})$, we have $\alpha_{g}=h(\mathbf{s}) \alpha_{g}^{1}$ and $\alpha_{s k}=\alpha_{s k}^{1}$.

Proof: The transform between $u_{j}=g^{-1} u_{\mathrm{f} j}$ and $\left(\alpha_{g}, \alpha_{s}\right)$ is $\alpha_{g}=\sum_{j=1}^{N-1} \rho_{j} \times u_{j}$ and $\alpha_{s k}=\sum_{j=1}^{N-1}\left(\frac{\partial \rho_{j}}{\partial s_{k}}-A_{k} \times \rho_{j}\right)^{\top} u_{j}$. By applying Lemma 2.2 , it is straightforward to show that $\frac{\partial \rho_{j}}{\partial s_{k}}-A_{k} \times$ $\rho_{j}=h(\mathbf{s})\left(\frac{\partial \rho_{j}^{1}}{\partial s_{k}}-A_{k}^{1} \times \rho_{j}^{1}\right)$. On the other hand, $u_{j}$ are gauge covariant because $u_{j}=g^{-1} u_{\mathrm{f} j}=h(\mathbf{s})\left(g^{1}\right)^{-1} u_{\mathrm{f} j}=h(\mathbf{s}) u_{j}^{1}$. Then $\alpha_{g}=h(\mathbf{s}) \sum_{j=1}^{N-1} \rho_{j}^{1} \times u_{j}^{1}=h(\mathbf{s}) \alpha_{g}^{1}$ and $\alpha_{s k}=\sum_{j=1}^{N-1}\left(\frac{\partial \rho_{j}^{1}}{\partial s_{k}}-A_{k}^{1} \times\right.$ $\left.\rho_{j}^{1}\right)^{\top} h^{\top}(\mathbf{s}) h(\mathbf{s}) u_{j}^{1}=\alpha_{s k}^{1}$.

\section{Freedom of Choosing Shape Coordinates}

With the the gauge covariant control defined, we present special control forms that further allow each particle freedom of choosing shape coordinates. Each particle selects its own set of Jacobi shape coordinates and does not necessarily know about choices made by other particles. The control forces computed by each particle are consistent with other particles.

Consider the Jacobi shape space $B$ that is a smooth manifold. Let $\mathbf{s}^{1}$ and $\mathbf{s}^{2}$ be two sets of shape coordinates on a coordinate patch. Suppose that for any point in this coordinate patch, the coordinate transform $\mathbf{s}^{1}=\mathbf{s}^{1}\left(\mathbf{s}^{2}\right)$ is a bijection. Define the matrix $\mathbf{J}=\frac{\partial \mathbf{s}^{1}}{\partial \mathbf{s}^{2}}$ with its $k, l$-th element $J^{k l}$. Then $\mathbf{J}$ is a $(3 N-6) \times(3 N-6)$ square matrix with full rank.

Furthermore, consider two sets of Jacobi vectors that describe the same formation. According to the democracy property of Jacobi vectors, there exists $\mathbf{h} \in \mathrm{O}(\mathrm{N}-1)$ s.t.

$$
\left[\begin{array}{llll}
\rho_{\mathrm{f} 1}^{1} & \rho_{\mathrm{f} 2}^{1} & \ldots & \rho_{\mathrm{f}(N-1)}^{1}
\end{array}\right]=\left[\begin{array}{lllll}
\rho_{\mathrm{f} 1}^{2} & \rho_{\mathrm{f} 2}^{2} & \ldots & \rho_{\mathrm{f}(N-1)}^{2}
\end{array}\right] \mathbf{h} .
$$

Consider the gauge transform $g^{1}(\mathbf{s})=g^{2}\left(\mathbf{s}^{2}\right) h^{\top}(\mathbf{s})$ where $h(\mathbf{s})$ represents $h^{1}\left(\mathbf{s}^{1}\right)=h^{2}\left(\mathbf{s}^{2}\right)$. Under such gauge transform we have $g^{1}\left[\begin{array}{llll}\rho_{1}^{1} & \rho_{2}^{1} & \ldots & \rho_{(N-1)}^{1}\end{array}\right]=g^{2}\left[\begin{array}{llll}\rho_{1}^{2} & \rho_{2}^{2} & \ldots & \rho_{(N-1)}^{2}\end{array}\right]$ h. This can be written as

$$
\rho_{j}^{1}=h(\mathbf{s}) \sum_{i=1}^{N-1} \mathbf{h}_{i j} \rho_{i}^{2} .
$$

Lemma 3.3: Consider the shape coordinate transform $\mathbf{s}^{1}=\mathbf{s}^{1}\left(\mathbf{s}^{2}\right)$, the gauge transform $g^{1}\left(\mathbf{s}^{1}\right)=g^{2}\left(\mathbf{s}^{2}\right) h^{\top}(\mathbf{s})$, and the change of Jacobi vectors given by equation (14). We have, for $k=1,2, \ldots, 3 N-6$ and $j=1,2, \ldots, N-1$,

$$
\begin{gathered}
\frac{\partial \rho_{j}^{1}}{\partial s_{k}^{1}}=h(\mathbf{s}) \sum_{i=1}^{N-1} \mathbf{h}_{i j}\left(\sum_{l=1}^{3 N-6} J^{k l}\left(\frac{\partial \rho_{i}^{2}}{\partial s_{l}^{2}}+\widehat{\gamma}_{l}^{2} \rho_{i}^{2}\right)\right), \\
A_{k}=h(\mathbf{s}) \sum_{l=1}^{3 N-6} J^{k l}\left(A_{l}^{1}+\gamma_{l}^{1}\right),
\end{gathered}
$$


and

$$
I^{1}\left(\mathbf{s}^{1}\right)=h(\mathbf{s}) I^{2}\left(\mathbf{s}^{2}\right) h^{\top}(\mathbf{s})
$$

The proof is omitted since it can be carried out in a similar way as the proofs of Lemma 2.1 and Lemma 3.2. The following theorem states that if certain rules are followed, then the control forces computed in a distributed fashion by different particles are consistent under the gauge transform and shape coordinate transform.

Theorem 3.4: Let particle 1 and particle 2 be two members of a $\mathrm{N}$-particle formation governed by the controlled Lagrange equations (11) and (12). To describe the formation, consider the case that the two particles have established two gauge conventions, selected two sets of Jacobi vectors, and chosen two sets of shape coordinates that are connected by the gauge transform, the changes of Jacobi vectors, and the shape coordinate transform as in Lemma 3.3. Suppose the feedback control $\left(\alpha_{g}^{1}, \alpha_{s}^{1}\right)$ computed by particle 1 and $\left(\alpha_{g}^{2}, \alpha_{s}^{2}\right)$ computed by particle 2 are functions of gauge invariant and gauge covariant quantities and satisfy, for $k=1,2, \ldots, 3 N-6$,

$$
\begin{aligned}
\alpha_{g}^{1} & =h(\mathbf{s}) \alpha_{g}^{2} \\
\left(\alpha_{s}^{1}\right)_{k} & =\sum_{l=1}^{3 N-6} J^{k l}\left(\alpha_{s}^{2}\right)_{l} .
\end{aligned}
$$

Suppose the two particles use the same control force $u_{c}$ for the center of the formation. Then the forces applied to each particle computed by particle 1 are identical to the forces applied to each particle computed by particle 2 when compared in the inertial frame i.e. $\mathbf{f}_{i}^{1}=\mathbf{f}_{i}^{2}$ for $i=1,2, \ldots N$.

Proof: From the definition of $\left(\alpha_{g}, \alpha_{s}\right)$, we have the following equations

$$
\begin{aligned}
& \sum_{j=1}^{N-1} \rho_{j}^{1} \times u_{j}^{1}=h(\mathbf{s}) \sum_{j=1}^{N-1} \rho_{j}^{2} \times u_{j}^{2} \\
& \sum_{j=1}^{N-1}\left(\frac{\partial \rho_{j}^{1}}{\partial s_{k}}-A_{k}^{1} \times \rho_{j}^{1}\right)^{\top} u_{j}^{1}=\sum_{l=1}^{3 N-6} J^{k l} \sum_{j=1}^{N-1}\left(\frac{\partial \rho_{j}^{2}}{\partial s_{l}}-A_{l}^{2} \times \rho_{j}^{2}\right)^{\top} u_{j}^{2}(20)
\end{aligned}
$$

It is straightforward to show that

$$
\sum_{j=1}^{N-1} \rho_{j}^{1} \times u_{j}^{1}=h(\mathbf{s})\left(\sum_{i=1}^{N-1} \rho_{i}^{2} \times\left(\sum_{j=1}^{N-1} \mathbf{h}_{i j} h^{\top}(\mathbf{s}) u_{j}^{1}\right)\right) .
$$

Now apply Lemma 3.3, we have

$$
\begin{aligned}
& \sum_{j=1}^{N-1}\left(\frac{\partial \rho_{j}^{1}}{\partial s_{k}}-A_{k}^{1} \times \rho_{j}^{1}\right)^{\top} u_{j}^{1} \\
& =\sum_{l=1}^{3 N-6} J^{k l} \sum_{i=1}^{N-1}\left(\left(\frac{\partial \rho_{i}^{2}}{\partial s_{l}}-A_{l}^{2} \times \rho_{i}^{2}\right)^{\top}\left(\sum_{j=1}^{N-1} \mathbf{h}_{i j} h^{\top}(\mathbf{s}) u_{j}^{1}\right)\right) .
\end{aligned}
$$

Equations (21) and (22) imply that $u_{j}^{2}=h^{\top}(\mathbf{s}) \sum_{i=1}^{N-1} \mathbf{h}_{j i} u_{i}^{1}$, which can be written in the following form: $\left[\begin{array}{llll}u_{1}^{2} & u_{2}^{2} & \ldots & u_{(N-1)}^{2}\end{array}\right]=$ $h^{\top}(\mathbf{s})\left[\begin{array}{llll}u_{1}^{1} & u_{2}^{1} & \ldots & u_{(N-1)}^{1}\end{array}\right] \mathbf{h}^{\top}$. Therefore, the controls $u_{\mathrm{f} j}^{1}$ and $u_{\mathrm{f} j}^{2}$ for the Jacobi vectors in the inertial frame satisfy $\left[\begin{array}{llll}u_{\mathrm{f} 1}^{1} & u_{\mathrm{f} 2}^{1} & \ldots & u_{\mathrm{f}(N-1)}^{1}\end{array}\right]=$ $\left[\begin{array}{llll}u_{\mathrm{f} 1}^{2} & u_{\mathrm{f} 2}^{2} & \ldots & u_{\mathrm{f}(N-1)}^{2}\end{array}\right] \mathbf{h}$. Then given $u_{c}^{1}=u_{c}^{2}$, this implies that $\mathbf{f}_{i}^{1}=\mathbf{f}_{i}^{2}$ for $i=1,2, \ldots N$.

\section{Formation Control and Shape Consensus}

In this section, we design gauge covariant formation control laws and consensus algorithms that use $\Upsilon, \mathbf{s}$, and $\dot{\mathbf{s}}$ as feedback to achieve constant shape with constant rotation. We assume that robots are able to measure these quantities with sensors mounted onboard. This assumption is reasonable in practice since a wide variety of range sensors, angle sensors, and stargazers are available on modern mobile robots.
We start with a general Lyapunov candidate function on the Jacobi pre-shape space as

$$
V=K+\Delta(\mathbf{s})+\frac{1}{2} \Upsilon_{0}^{\top} \Upsilon_{0}-\Upsilon_{0}^{\top} \Upsilon
$$

where the $K$ is the kinetic energy given by (2) and $\Upsilon_{0}$ specifies a desired gauge covariant angular velocity. The function $\Delta(\mathbf{s}) \geq 0$ is a continuously differentiable function such that $\Delta\left(\mathbf{s}_{0}\right)=0$ where $\mathbf{s}_{0}$ specifies a desired shape. We also require that $\Delta^{\prime}\left(\mathbf{s}_{0}\right)=0$ if and only if $\mathbf{s}=\mathbf{s}_{0}$. An equivalent form of the function $V$ is $V=\Delta(\mathbf{s})+$ $\frac{1}{2}\left(\Upsilon-\Upsilon_{0}\right)^{\top} I\left(\Upsilon-\Upsilon_{0}\right)+\frac{1}{2} \dot{\mathbf{s}}^{\top} G \dot{\mathbf{s}}$, which clearly shows that $V$ is positive definite.

We follow the procedure of a Lyapunov function based design. By the balance law between work and energy [17] or by direct calculation, the time derivative of $K$ along the controlled dynamics (11) and (12) is $\dot{K}=\left\langle\Omega, u_{g}\right\rangle+\left\langle\dot{\mathbf{s}}, u_{s}\right\rangle$. The time derivative of $V$ is then

$$
\begin{aligned}
\dot{V} & =\left\langle\dot{\mathbf{s}}, u_{s}+\Delta^{\prime}(\mathbf{s})+\frac{1}{2}\left(\frac{\partial I}{\partial \mathbf{s}}\right)^{*}\left[\Upsilon_{0}, \Upsilon_{0}\right]_{\tau}\right\rangle+\left\langle\Omega-\Upsilon_{0}, u_{g}\right\rangle \\
& +\left\langle\Upsilon_{0}, \Omega \times \Pi\right\rangle .
\end{aligned}
$$

The last term can be re-arranged using the fact that $\left\langle\Upsilon_{0}, \Upsilon_{0} \times \Pi\right\rangle=0$ i.e. $\left\langle\Upsilon_{0}, \Omega \times \Pi\right\rangle=\left\langle\Upsilon_{0},\left(\Omega-\Upsilon_{0}\right) \times \Pi\right\rangle=\left\langle\left(\Omega-\Upsilon_{0}\right), \Pi \times \Upsilon_{0}\right\rangle$. Therefore, the time derivative of $V$ is finally

$$
\dot{V}=\left\langle\dot{\mathbf{s}}, u_{s}+\Delta^{\prime}(\mathbf{s})+\frac{1}{2}\left(\frac{\partial I}{\partial \mathbf{s}}\right)^{*}\left[\Upsilon_{0}, \Upsilon_{0}\right]_{\tau}\right\rangle+\left\langle\Omega-\Upsilon_{0}, u_{g}-\Upsilon_{0} \times I\right\rangle .
$$

We design a cooperative control law to be

$$
\begin{aligned}
& \alpha_{g}=\Upsilon_{0} \times I-k_{1}\left(\Upsilon-\Upsilon_{0}\right) \\
& \alpha_{s}=-\Delta^{\prime}(\mathbf{s})-\frac{1}{2}\left(\frac{\partial I}{\partial \mathbf{s}}\right)^{*}\left[\Upsilon_{0}, \Upsilon_{0}\right]_{\tau}-k_{1} \dot{\mathbf{s}}
\end{aligned}
$$

where $k_{1}>0$ is a constant gain. This control law is gauge covariant. After using (13) to compute $\left(u_{g}, u_{s}\right)$, we can show that

$\dot{V}=-k_{1}\|\dot{\mathbf{s}}\|^{2}-k_{1}\left\|\Upsilon-\Upsilon_{0}\right\|^{2} \leq 0$ with $\dot{V}=0$ if and only if $\Upsilon=\Upsilon_{0}$ and $\dot{\mathbf{s}}=0$. We are able to prove the following theorem.

Theorem 4.1: The gauge covariant cooperative feedback control law (26) locally stabilizes the Jacobi shape $\mathbf{s}_{0}$ and the gauge covariant angular velocity $\Upsilon_{0}$ asymptotically.

We omit the proof here since it follows the standard procedure of convergence proof applying LaSalle's invariance principle. We note the following:

Shape Consensus: Suppose we select $\Delta(\mathbf{s})=0$ for all $\mathbf{s}$ and $\Upsilon_{0}=0$. Then $\alpha_{g}=-k_{1} \Upsilon$ and $\alpha_{s}=-k_{1} \dot{\mathbf{s}}$. When subjected to the gauge transform and shape coordinate transform as in Lemma 3.3, the conditions in (19) are satisfied. Therefore, according to Theorem 3.4, we have obtained a distributed shape consensus algorithm that allows each particle to select its own body frame and shape coordinates. The formation will stop rotation and the shape $\mathbf{s}$ will converge to constant values. This implies that shape consensus is achieved among the particles.

Coordinate Free Formation Control: Suppose we select $\Delta(\mathbf{s})=$ $\frac{1}{2} \sum_{j=1}^{N-1}\left\|\rho_{j}(\mathbf{s})-\rho_{j}\left(\mathbf{s}_{0}\right)\right\|$. One can verify that $\Delta^{\prime}(\mathbf{s})=0$ if and only if $\mathbf{s}=\mathbf{s}_{0}$. Furthermore, it is straightforward to show that this $\Delta^{\prime}(\mathbf{s})$ term makes the control laws in equation (26) satisfy the conditions in (19). Therefore, the formation control law can be implemented in a distributed manner, allowing each particle freedom to choose body frame and shape coordinates.

\section{Conclusions}

In this paper we introduce a geometric approach based on Jacobi shape theory to study formation dynamics and control. Our results show that gauge invariance and covariance can be utilized to allow the freedom of choice of body frames and shape coordinates for 
each robot. Such freedom enables distributed computation of the control laws as well as shape consensus algorithms. We develop distributed gauge covariant feedback control achieving constant shape and rotation of the formation with provable convergence.

\section{REFERENCES}

[1] R. Olfati-Saber, J. A. Fax, and R. M. Murray, "Consensus and cooperation in networked multi-agent systems," Proceedings of the IEEE, vol. 95, no. 1, pp. 215-233, 2007.

[2] W. Ren and R. W. Beard, Distributed Consensus in Multi-vehicle Cooperative Control. Springer-Verlag, 2008.

[3] A. Jadbabaie, J. Lin, and A. S. Morse, "Coordination of groups of mobile agents using nearest neighbor rules," IEEE Trans. on Automatic Control, vol. 48, no. 6, pp. 988-1001, 2003.

[4] R. Olfati-Saber and R. M. Murray, "Consensus problems in networks of agents with switching topology and time-delays," IEEE Trans. on Automatic Control, vol. 49, no. 9, pp. 1520-1533, 2004.

[5] L. Moreau, "Stability of multiagent systems with time-dependent communication links," IEEE Transactions on Automatic Control, vol. 50, no. 2, pp. 169-182, 2005.

[6] J. Cortés, S. Martínez, and F. Bullo, "Robust rendezvous for mobile autonomous agents via proximity graphs in arbitrary dimensions," IEEE Transactions on Automatic Control, vol. 51, no. 8, pp. 1289-1298, 2006.

[7] R. Littlejohn and M. Reinsch, "Gauge fields in the separation of rotation and internal motions in the n-body problem," Reviews of Modern Physics, vol. 69, no. 1, pp. 213-275, 1997.

[8] F. Zhang, M. Goldgeier, and P. S. Krishnaprasad, "Control of small formations using shape coordinates," in Proc. of 2003 International Conf. of Robotics and Automation. Taipei, Taiwan: IEEE, 2003, pp. 25102515.

[9] F. Zhang, "Cooperative shape control of particle formations," in Proc. of 46th IEEE Conf. on Decision and Control, New Orleans, LA, 2007, pp. 2516-2521.

[10] C. Belta and V. Kumar, "Abstraction and control for groups of robots," IEEE Trans. on Robotics, vol. 20, no. 5, pp. 865-875, 2004.

[11] J. Derenick and J. Spletzer, "Convex optimization strategies for coordinating large-scale robot formations," IEEE Trans. Robotics, vol. 23, no. 6, pp. 1252-1259, 2007.

[12] A. Bloch, J. Baillieul, P. Crouch, and J. E. Marsden, Nonholonomic Mechanics and Control. Springer, 2003.

[13] R. Abraham and J. Marsden, Foundations of Mechanics, 2nd Ed. Addison-Wesley, 1978.

[14] L.-S. Wang, "Geometry, dynamics and control of coupled systems," $\mathrm{Ph}$.D. dissertation, ISR Technical Report PhD90-5, University of Maryland, 1990.

[15] L.-S. Wang and P. S. Krishnaprasad, "Gyroscopic control and stabilization," Journal of Nonlinear Science, vol. 2, pp. 367-415, 1992.

[16] S. S. Antman, Nonlinear problems of elasticity. New York: SpringerVerlag, 1995.

[17] H. Goldstein, C. Poole, and J. Safko, Classical Mechanics, 3rd Ed. Addison-Wesley, 2002. 